\title{
The influence of denoising on the subgrade settlement fitting curve
}

\author{
Zhangjun DAl ${ }^{\mathrm{a}}$, Shanxiong $\mathrm{CHEN}^{\mathrm{b}}$
}

State Key Laboratory of Geomechanics and Geotechnical Engineering, Institute of Rock and Soil Mechanics, Chinese Academy of Sciences, Wuhan 430071, China

azjdai@whrsm.ac.cn, bsxchen@whrsm.ac.cn

Keywords: wavelet; denoising; subgrade; settlement; correlation coefficient

Abstract. High speed railway must strictly control the settlement deformation. It has important significance to explore reasonable methods of noise reduction for the settlement curve, in order to improve the reliability of settlement prediction. Wavelet analysis is feasible to denoise the observation data. The development trend of settlement curve before and after denoising is consistent. While removing the noise from the original data, the validity of the original data is preserved. Wavelet denoising can improve the regression correlation coefficient of the settlement curve.

\section{Introduction}

High speed railway must strictly control the settlement and deformation of the structure, especially the post-construction settlement. It has important practical significance to calculate the settlement by using the settlement observation data[1,2].

In the actual settlement observation, the improve of the monitoring accuracy is limited, it is vulnerable to the interference of external conditions, especially late in the settlement monitoring, the deformation is small in a short period of time, maybe a weak signal, but the error is presented for strong signal, the deformation trend is likely to be submerged in noise and cannot be identified. According to the specifications, the observed data should be analyzed with curve regression, the correlation coefficient should not be less than $0.92[3,4]$. Therefore, the extraction of useful information from the irregular settlement monitoring data, analysis of the deformation law is one of the key contents of the deformation analysis[5].

Wavelet analysis is a high performance signal analysis method, which has good performances in data filtering, signal feature extraction and noise reduction of the settlement monitoring data[6,7]. For the passenger dedicated line settlement data, through the wavelet analysis, exploring reasonable methods of noise reduction for the settlement curve, in order to improve the reliability of settlement prediction, it has important significance in engineering.

\section{Settlement observation data feature of high-speed railway}

It has strict control standards on post-construction settlement and differential settlement for offline roadbed, bridges and culverts, tunnels and other projects of High-speed railway, the post-construction settlement in full line must be less than $15 \mathrm{~mm}$, the limits of the ballastless track adjustment. In order to meet the requirements, a variety of measures such as CFG pile, compaction, grouting and jet grouting piles are used on the original foundation reinforcement, which determines the small magnitude of the settlement and the high accuracy of the settlement measurement. In Hubei, the measurement accuracy of settlement is $\pm 1 \mathrm{~mm}$, and readings to $0.1 \mathrm{~mm}$.

It can be seen from the current settlement observation data in Hubei intercity railway, except for some soft soil subgrade by preloading segments, the foundation deformation can reach 40 50mm, most of the subgrade settlement deformation is less than $15 \mathrm{~mm}$, and it is less than $5 \mathrm{~mm}$ for bridges and tunnels, that is to say, the magnitude of settlement is small in Hubei intercity railway.Since the magnitude of settlement is small, and the settlement increments is smaller after loading. The settlement system error caused by the restriction of technical conditions and the settlement increment are substantially in the same order of magnitude, a small measurement error will cause a great fluctuation in the observed data. Thus, the settlement observation data fluctuations increased the difficulties for 
settlement prediction and evaluation. Existing observational data reflects the relative fluctuation is obvious for small deformation observation data.

\section{The characteristics and description method of subgrade settlement curve}

A large number of engineering practices have proved that, the curve of foundation settlement development with the time can be approximately described as hyperbola or exponential. In order to simulate the actual settlement process of high-speed railway subgrade, a set of data satisfies the Eq.1 is selected as an ideal noise-free settlement curve for the simulation.

$$
\begin{aligned}
& S_{t}=S_{0}+t /(a+b t) \\
& S_{f}=S_{0}+1 / b
\end{aligned}
$$

where, $S_{t}$ is the settlement in time $t, S_{f}$ and $S_{0}$ are the final and initial settlement, $a, b$ are coefficients.

The hyperbolic method assumes that the initial settlement is zero, $S_{0}=0$. In order to simulate the settlement curve contained noise, a random parameter is added behind the hyperbolic equation.

$$
S=t /(a+b t)+\operatorname{cr}(x)
$$

Where, $S$ is the simulate settlement, $r(x)$ is a random function, $c$ is a parameter to control the wave amplitude of the random function. As can be seen from the equation, $a$ controls the convergence speed of the simulate settlement curve, $b$ controls the cumulative settlement, $c$ controls the fluctuations.

\section{Wavelet denoising of the observation results of the subgrade settlement}

\section{The basic theory of wavelet transform}

A wavelet function, translation and scaling factors are $a$ and $b$, Eq. 4 is the definition of wavelet base.

$$
\psi_{a, b}(t)=|a|^{-1 / 2} \psi[(t-b) / a] \quad a, b \in R \text { and } a \neq 0
$$

For any function or signal $f(t) \in L^{2}(R)\left(L^{2}(R)\right.$ is the real space of square integrable), the wavelet transform is the inner product of this function and wavelet function :

$$
W_{f}(a, b)=\left\langle f(t), \psi_{a, b}(t)\right\rangle=|a|^{-1 / 2} \int_{R} f(t) \bar{\psi}[(t-b) / a] d t
$$

The parameters $a$ and $b$ should be discretized.

$$
a=a_{0}^{j}, b=k b_{0} a_{0}^{j},\left(a>1, b_{0} \in R, j / k \in Z\right)
$$

Put them into the refoot definition equation, get the discrete wavelet function.

$$
\psi_{j, k}(t)=a_{0}^{-j / 2} \psi\left[\left(t-k b_{0} a_{0}^{j}\right) / a_{0}^{j}\right]=a_{0}^{-j / 2} \psi\left(a_{0}^{-j} t-k b_{0}\right)
$$

It is possible to find the wavelet transform of the real valued function $f(t)$.

$$
D_{f}(j, k)=\left\langle f(t), \psi_{j, k}(t)\right\rangle=a_{0}^{-j / 2} \int_{R} f(t) \psi\left(a_{0}^{-j} t-k b_{0}\right) d t
$$

When $a_{0}=2, b_{0}=1$, the two equations become discrete dyadic wavelet and its transform style, then,

$$
\begin{aligned}
& \psi_{j, k}(t)=2^{-j / 2} \psi\left(2^{-j} t-k\right) \\
& D_{f}(j, k)=2^{-j / 2} \int_{R} f(t) \psi\left(2^{-j} t-k\right) d t
\end{aligned}
$$

Therefore, the choice of wavelet function is equivalent to choose a wavelet basis. According to the definition of wavelet function, wavelet function $\psi(t)$ is diversity. Therefore, it is very important to select an appropriate wavelet function in the practical application.

\section{The application of wavelet transform in the analysis of the observation data}

Usually think of the observed data $x(t)$ consists two parts, the useful signal $s(t)$ and random noise $n(t)$. The useful signal $s(t)$ may be the actual observation signal or the deterministic noise, and may be both.

$$
x(t)=s(t)+n(t), n(t) \square N\left(0, \sigma^{2}\right)
$$

Using wavelet transform for denoising the original observation data, the analysis steps are as follows: (1) Wavelet decomposition, (2) Wavelet threshold quantization, (3) Wavelet reconstruction. 
This sample is $a=4, b=0.1\left(S_{f}=10 \mathrm{~mm}\right), c=1$ (the fluctuations value between $\pm 1 \mathrm{~mm}$ ), get the simulated settlement curve contained noise, using wavelet analysis toolbox in Matlab, to denoising the settlement observation data of one year observation period after the main project completion, Using Sym8 wavelet to decompose the signal, in the fifth layer of the decomposition, the value selection method of signal denoising based on heuristic SURE domain, use heuristic SURE threshold selection method to denoising the signal. The denoising result is shown in Fig.1.

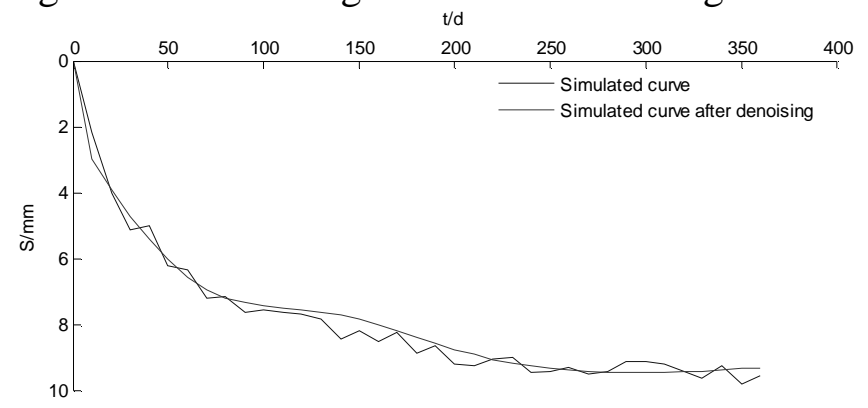

\section{Figure 1 The denoising result of the simulated settlement curve}

\section{The influence of denoising on the fitting correlation coefficient of the settlement curve}

\section{Simulation plan}

According to the actual engineering, $a$ is generally less than 40 (a high value of $a$ causes a linear curve failed to reach the prediction standard), the predicted $S_{f}$ is generally less than $100 \mathrm{~mm}$, and the fluctuation range $c$ is within $3 \mathrm{~mm}$. Take the most common situation as examples, on this basis, to explore the denoising effect to the curve fitting correlation coefficient when $a, b, c$ change.

$a$ is respectively $40,27,4,1.4,1,0.8$ and $0.4 . b$ is respectively $0.2,0.1,0.05,0.033,0.017$ and 0.01 ( $S_{f}$ is respectively $5,10,20,30,60$ and 100). $c$ is respectively $0.5,1,1.5,2$ and 3 . It is assumed the observation period is one year after the completion of the main project in the simulated curves.

\section{$a$ is a variable}

When $\mathrm{b}=0.1, c=1$, adjusted $a$, using hyperbola method for curve fitting, the regression curves are shown in Fig. 2, the correlation coefficient $R$ before and after denoising with the changes of $a$ are listed in Table 1.
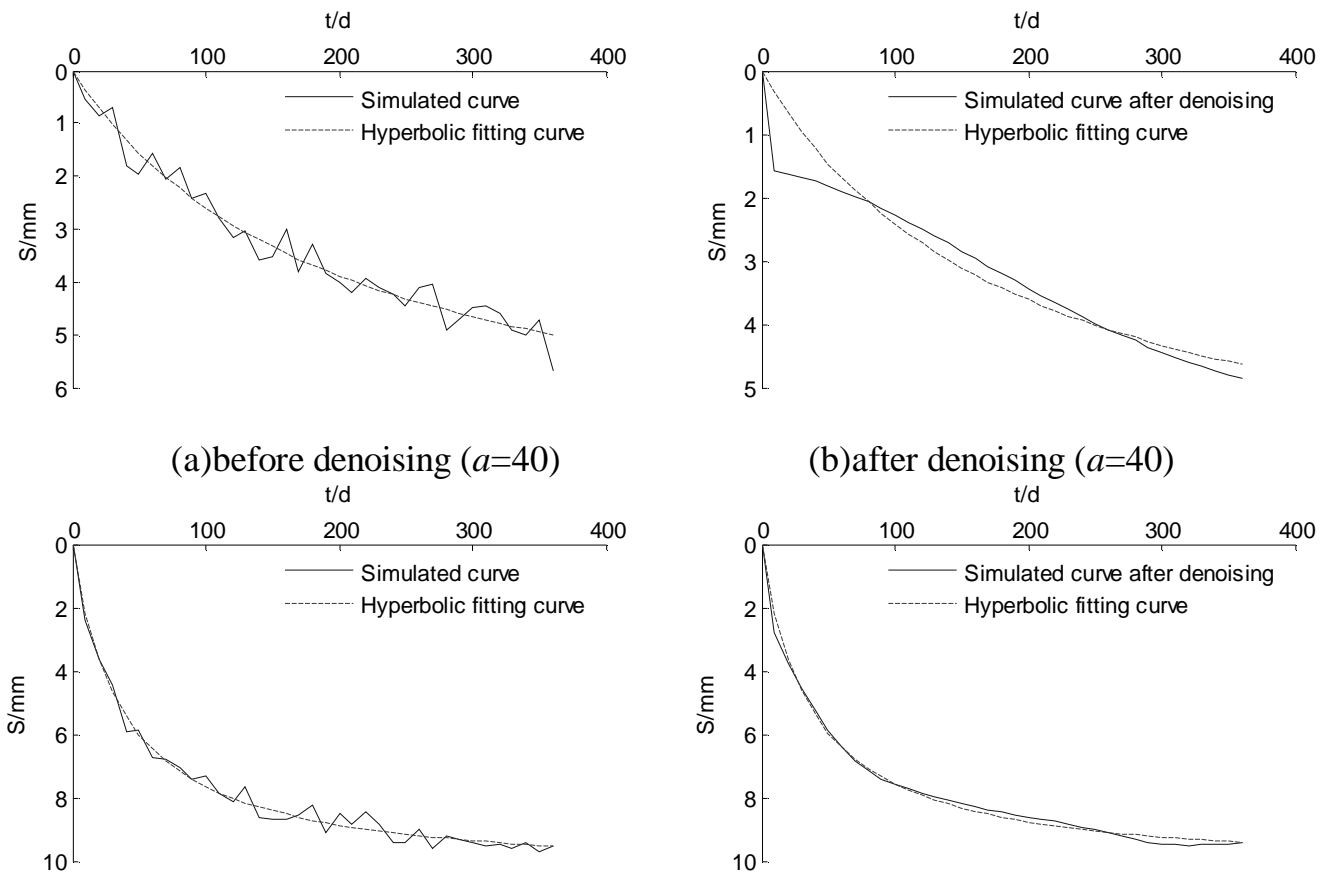

(c)before denoising $(a=4)$

(d)after denoising $(a=4)$

Figure 2 The hyperbolic fitting curves before and after denoising when $a$ changes 
Table 1 The change of the correlation coefficient $R$ when $a$ changes

\begin{tabular}{c|c|c|c||c|c|c|c}
\hline$a$ & $\begin{array}{c}\text { Before } \\
\text { denoising } R\end{array}$ & $\begin{array}{c}\text { After denoising } \\
R^{\prime}\end{array}$ & Increase of $R / \%$ & $a$ & $\begin{array}{c}\text { Before } \\
\text { denoising } R\end{array}$ & $\begin{array}{c}\text { After denoising } \\
R^{\prime}\end{array}$ & Increase of $R / \%$ \\
\hline 40 & 0.9753 & 0.9960 & 2.12 & 1 & 0.9819 & 0.9983 & 1.67 \\
\hline 27 & 0.9846 & 0.9972 & 1.28 & 0.8 & 0.9774 & 0.9964 & 1.94 \\
\hline 4 & 0.9853 & 0.9865 & 0.12 & 0.4 & 0.9722 & 0.9865 & 1.47 \\
\hline 1.4 & 0.9834 & 0.9960 & 1.28 & & & & \\
\hline
\end{tabular}

When $b, c$ are fixed, $a$ decreases, before denoising, the correlation coefficient of the regression curve increases first and then decreases, but overall the values remain large. And after denoising, the correlation coefficient is basically stable and larger.

\section{$b$ is a variable}

When $a=4, c=1$, adjusted $b$, using hyperbola method for simulation curve fitting, the regression curves are shown in Fig. 3, the correlation coefficient $R$ before and after denoising with the changes of $b$ are listed in Table 2.

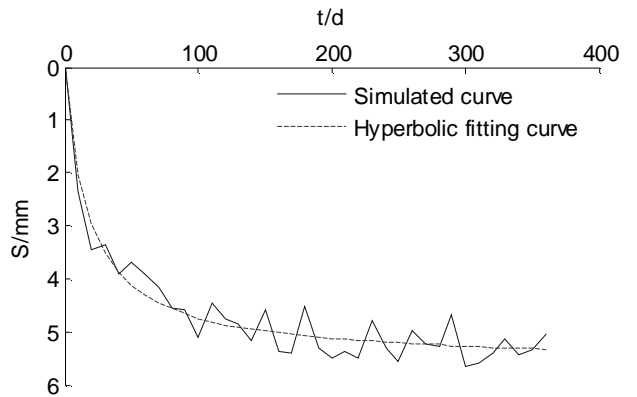

(a)before denoising $(b=0.2)$

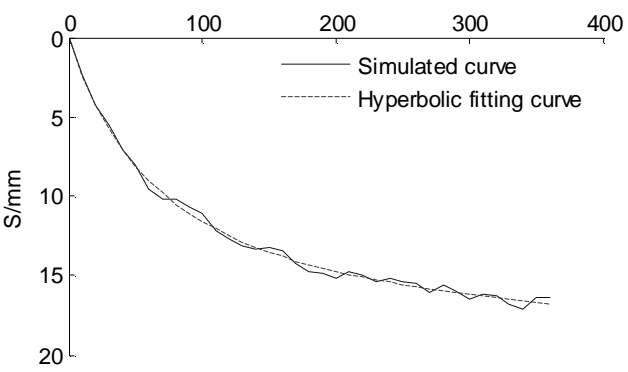

(c)before denoising $(b=0.05)$

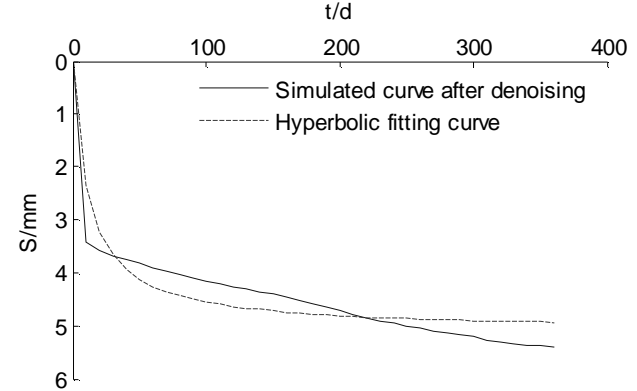

(b)after denoising $(b=0.2)$

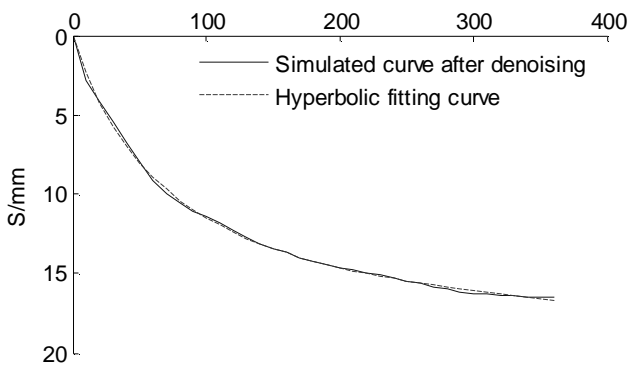

(d)after denoising $(b=0.05)$

Figure 3 The hyperbolic fitting curves before and after denoising when $b$ changes Table 2 The change of the correlation coefficient $R$ when $b$ changes

\begin{tabular}{c|c|c|c|c||c|c|c|c|c}
\hline$b$ & $\begin{array}{c}S_{f} / \\
\mathrm{mm}\end{array}$ & $\begin{array}{c}\text { Before } \\
\text { denoising } R\end{array}$ & $\begin{array}{c}\text { After } \\
\text { denoising } R\end{array}$ & $\begin{array}{c}\text { Increase of } \\
R / \%\end{array}$ & $b$ & $\begin{array}{c}S_{f} / \\
\mathrm{mm}\end{array}$ & $\begin{array}{c}\text { Before } \\
\text { denoising } R\end{array}$ & $\begin{array}{c}\text { After } \\
\text { denoising } R\end{array}$ & $\begin{array}{c}\text { Increase of } \\
R / \%\end{array}$ \\
\hline 0.2 & 5 & 0.9559 & 0.9591 & 0.33 & 0.033 & 30 & 0.9999 & 1.0000 & 0.01 \\
\hline 0.1 & 10 & 0.9853 & 0.9865 & 0.12 & 0.017 & 60 & 0.9999 & 1.0000 & 0.01 \\
\hline 0.05 & 20 & 0.9969 & 0.9973 & 0.04 & 0.01 & 100 & 0.9999 & 1.0000 & 0.01 \\
\hline
\end{tabular}

When $a, c$ are fixed, $b$ decreases, before denoising, the correlation coefficient of the regression curve increases, and overall the values are large. When $b$ is large, after denoising, the increase of the correlation coefficient is relatively larger, when $b$ is small, the correlation coefficient of the fitting curve is always one.

\section{$c$ is a variable}

When $a=4, b=0.1, S_{f}=10 \mathrm{~mm}$, adjusted $c$, using hyperbola method for simulation curve fitting, the regression curves are shown in Fig. 4, the correlation coefficient $R$ before and after denoising with the changes of $c$ are listed in Table 3. 


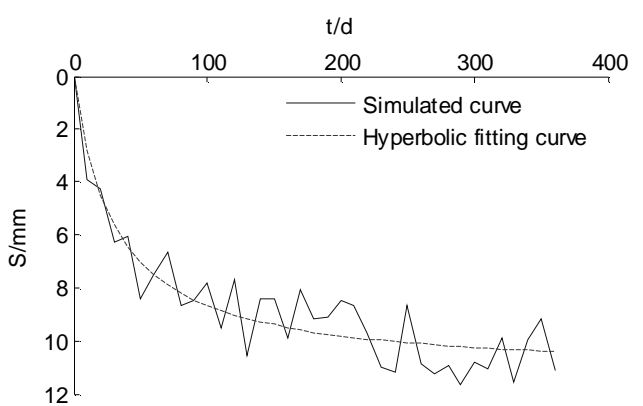

(a)before denoising $(c=3)$

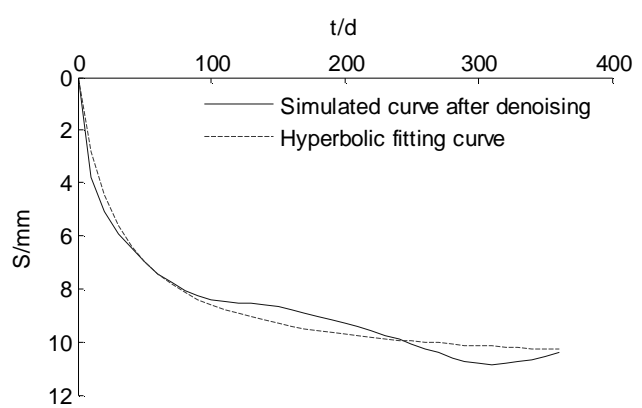

(b)after denoising $(c=3)$

Figure 4 The hyperbolic fitting curves before and after denoising when $c$ changes Table 3 The change of the correlation coefficient $R$ when $c$ changes

\begin{tabular}{c|c|c|c||c|c|c|c}
\hline$c$ & $\begin{array}{c}\text { Before denoising } \\
R\end{array}$ & $\begin{array}{c}\text { After denoising } \\
R^{\prime}\end{array}$ & $\begin{array}{c}\text { Increase of } R \\
/ \%\end{array}$ & $c$ & $\begin{array}{c}\text { Before denoising } \\
R\end{array}$ & $\begin{array}{c}\text { After denoising } \\
R^{\prime}\end{array}$ & $\begin{array}{c}\text { Increase of } R \\
/ \%\end{array}$ \\
\hline 0.5 & 0.9975 & 0.9893 & -0.82 & 2 & 0.9616 & 0.9883 & 2.78 \\
\hline 1 & 0.9853 & 0.9865 & 0.12 & 3 & 0.9258 & 0.9876 & 6.68 \\
\hline 1.5 & 0.9760 & 0.9891 & 1.34 & & & & \\
\hline
\end{tabular}

When $a, b$ are fixed, $c$ is small, curve fluctuation range is small, before and after denoising, the correlation coefficients of the regression curves are large, the effect of denoising is not significant. When $c$ is larger, the curve fluctuation is greater, wavelet denoising effect is more significant. When $c$ increases, the $R$ of the settlement curve decreases rapidly, but after denoising, the $R$ is generally more than 0.98 , which can meet the requirements of the specification.

\section{Conclusions}

It is feasible to use the method of wavelet analysis to denoise the observation data. The development trend of settlement curve before and after denoising is consistent. While removing the noise from the original data, the validity of the original data is preserved. When $a$ changes, wavelet denoising improves the regression correlation coefficient of the settlement curve. In the actual working conditions, $b$ is generally large, when $b$ changes, wavelet denoising improves the regression correlation coefficient of the settlement curve. When $c$ changes, the wavelet denoising has a significant impact on the regression coefficient of the settlement curve, the lager the $c$, the better effect of the denoising on the regression coefficient of the settlement curve.

\section{References}

[1] J Zhang, et al. Research on the Influence of Slant Level Ruler in the High-speed Railway Settlement Observation. Bulletin of Surveying \& Mapping 33.10(2011):47-46.

[2] D Y WU, Y P Yang and X. J. HU. Analysis of high-speed railway settlement observation data processing. Journal of Railway Science \& Engineering (2010).

[3] L F Jiang, et al. Settlement Deformation Evaluation Method of High-Speed Railway Cutting under Rebound Effect. Applied Mechanics \& Materials 300-301(2013):1081-1084.

[4] W B TU and Y F LI. Influence Analysis of Pile-slab Composite Foundation on the Settlement of High-speed Railway. Subgrade Engineering (2011).

[5] Z W Chen, et al. Safety threshold of high-speed railway pier settlement based on train track bridge dynamic interaction. Science China Technological Sciences 58.2(2015):202-210.

[6] J Y, HU, et al. Wavelet multi-scale Kalman filtering in settlement deformation for high-speed railway tunnel. Journal of Guilin University of Technology (2014).

[7] J M Wang. Research on the Application of Wavelet Threshold Denoising Method in High-speed Rail Settlement Observation Data Processing. Mine Surveying (2011). 